\title{
MODELING OF THE ELASTICALLY-MOUNTED CYLINDER BEHAVIOR IN THE CROSS-FLOW
}

\author{
SABINE UPNERE ${ }^{1,2 *}$ \\ ${ }^{1}$ Riga Technical University, Viskalu Street 36, Riga, Latvia \\ ${ }^{2}$ Ventspils University of Applied Sciences, Inzenieru Street 101, Ventspils, \\ Latvia
}

[Received: 16 August 2018. Accepted: 21 October 2019]

doi: 10.7546/JTAM.49.19.04.02

\begin{abstract}
This study investigates the Proper Orthogonal Decomposition (POD) combined with Radial Basis Functions (RBF) and Response Surface Method (RSM) based metamodels to appreciate the influence of the system parameters on elastically-mounted test cylinder (TC) behavior excited by water cross-flow. The TC mass, stiffness and damping coefficients and excitation forces are chosen as the design parameters. System responses at selected training points are obtained by full, two-dimensional model simulations using Computational Fluid Dynamics. Obtained results show that POD-RBF metamodel has good accuracy to predict the system parameters, which is not timedependent, whereas the RSM metamodel can reconstruct the time-dependent $\mathrm{TC}$ movement trajectory with high precision.
\end{abstract}

KEY WORDS: cross-flow, metamodeling, radial basis function, response surface method, proper orthogonal decomposition, vibration.

\section{INTRODUCTION}

The cross-flow-induced vibrations in tube arrays have been a significant influence on different engineering applications. The characteristics of tube responses on the flow are strongly dependent on the bundle geometry, the tube mechanical properties, the surrounding media, the flow velocity and other aspects. Therefore, every type of systems needs a separate investigation of the parameter impact. The full numerical modeling or physical experiments could be time-consuming and costly to do parametric studies.

The numerical models that can get the accurate input-output maps in online regime instead of computationally expensive Finite Element or Computational Fluid Dynamics (CFD) simulations have increasing importance in a lot of engineering fields. For example, model reduction methods are applied to predict the aerodynamic flow fields [1], to validate the ship maneuvering simulation models [2], to simulate gas

\footnotetext{
${ }^{*}$ Corresponding author e-mail: upnere@gmail.com
} 
explosions [3], in several geotechnical engineering fields [4,5] or to study vortex induced vibrations [6]. Metamodeling is one way how to obtain fast simulation tools for complex systems providing an accurate, low-cost function estimation. In this paper, the metamodeling is defined as a mathematical approximation to represent the characteristic behavior of the system and not to investigate the physical aspects of it.

A wide range of model reduction methods have been used to create metamodels, for example, Filho et al. [6] used a Kriging data fitting approximation to minimize the vortex-induced vibrations, the transfer matrix metamodel is introduced by Son and Savage [7] to work with time-varying input vectors, Braatz and Hisken [3] used Response Surface Method (RSM) to construct the gas explosion simulations. Metamodel approaches are convenient for optimization and inverse analysis problems, for example, Kato and Funazaki [8] applied Proper Orthogonal Decomposition (POD) combined with Radial Basis Functions (RBF's) to optimize the aerodynamic shape of the engine turbine, the wind-turbine optimization is done by Sessarego et al. [1], Hamim and Singh [9] used POD-RBF approach to do inverse analysis for identifying nonlinear burger model parameters. POD with Extended Radial Basis Function (POD-ERBF) which allows increase accuracy of metamodels for both linear and nonlinear problems is formulated by Khaledi et al. [4]. Metamodels can be implemented in applications for uncertainty quantification, reliability analysis, signal processing, system parameter estimation and many others.

In this paper, POD-RBF and RSM are used as methods to analyse the flowinduced vibrations in the tube bundle due to the water cross-flow. Metamodels are used to investigate the model parameter influence on the elastically-mounted test cylinder (TC) oscillation amplitudes and dominant frequencies as well to explore time-dependent excitation forces influence on the vibration trajectory of the TC. The mechanical properties of the TC are described by the mass, support stiffness and damping.

Large and small amplitude vibrations simulated by CFD were used to train the metamodels. The sample points are selected by Latin Hypercube sampling approach. Four radial basis functions were investigated to find the most appropriate for predictions of the TC behavior due to the flow-structure interaction. The second-order polynomial response surface approximation is applied to obtain the time-dependent fast simulation tool. The time-dependent metamodeling could be useful for the monitoring and diagnostics of the system.

\section{PROBLEM DESCRIPTION}

In this paper is explored a single, elastically-mounted cylinder (TC) behavior in turbulent water cross-flow. For simplicity, the $2 \mathrm{D}$ problem is analysed. The TC is placed in a closely-packed, fixed cylinder bundle with the triangular arrangement. The cylin- 
der bank is inserted in the rectangular channel. Mathematically the analysed TC motion due to the cross-flow can describe as the mass-spring-damper system:

$$
\mathbf{M} \ddot{q}+\mathbf{C} \dot{q}+\mathbf{K} q=Q
$$

where $\boldsymbol{q}$ is a vector of generalized coordinates $\left(\boldsymbol{q}^{\mathrm{T}}=\left[x_{1}, x_{2}\right]\right), \boldsymbol{Q}$ is a vector of generalized forces $\left(\boldsymbol{Q}^{\mathrm{T}}=\left[F_{1}, F_{2}\right]\right)$ and dot denotes time derivatives. Matrices $\mathbf{M}, \mathbf{C}$ and $\mathbf{K}$ contain the TC mass, damping and stiffness coefficients, respectively.

As excitation forces $Q$ are not known, the CFD approach is applied to numerically find the corresponding values of forces having in mind both the fluid and the structure impact.

There are developed two kinds of metamodels: static and time-dependent. For static case, the relationship between the TC mass, stiffness and damping and corresponding vibration amplitudes and frequencies are investigated. The time-dependent excitation force impact on the TC motion trajectory is studied with the transit case.

\section{The Proposed Methodology for Metamodeling}

The goal of the metamodeling is to find the fast simulation tool that should be used instead of time-consuming CFD simulations. As a model order reduction method, it is chosen POD and for responses function approximation is used RBF and RSM approaches.

\subsection{PROPER ORTHOGONAL DECOMPOSITION}

The POD is a robust approach of data processing to obtain a low-dimensional approximation of some high-dimensional problem over a domain of interest. The known input-output relationships of the system are used to make the model reduction.

The most frequently used POD methods are the Principal Component Analyses, the Karhunen-Loeve Decomposition and the Singular Value Decomposition (SVD). All these methods are refereeing to the same mathematics, and they are equivalent [10]. In this paper, the POD is realized by SVD which find the basis vectors collected in the matrix $\Phi$ satisfying the POD requirement in the sample space.

Using SVD the response matrix $\mathbf{Y}$ can decompose keeping only $K$ important singular values as

$$
\mathbf{Y}=\mathbf{V}_{K}^{1} \mathbf{S}_{K}\left(\mathbf{V}_{K}^{2}\right)^{\mathrm{T}}
$$

where $\mathbf{V}_{K}^{1}$ is the matrix of the left singular vectors, $\mathbf{V}_{K}^{2}$ is the matrix of the right singular vectors, and $\mathbf{S}_{K}$ is the matrix of the $K$ singular values. Based on [8], using the SVD method the matrix $\bar{\Phi}=\mathbf{V}_{K}^{1}$. 
The number of important singular values founds utilizing the coefficient of relative significance $p_{i}$ for each value [11]

$$
p_{i}=\frac{s_{i}^{2}}{\sum_{j=1}^{N} s_{j}^{2}}, \quad i=1, \ldots, N,
$$

where $N$ is a number of samples, $s$ is a singular value. The dominance of the singular values is determined by equalizing the sum of coefficients with some limit (4). In the present case, the limit is chosen to be 0.99 , and only one dominant singular value is used

$$
\sum_{i=1}^{K} p_{i}>0.99 .
$$

As mentioned by Son and Savage [7] the number of selected dominant singular values is only one of the possible cause of errors applying SVD method. The important role to reduce the error is the number of sampling points. In the time-dependent cases, the selected time step for input and responses functions has an impact on the prediction accuracy as well.

\subsection{RADIAL BASES FUNCTION}

RBF is used to construct an approximation of continuous function $f(\boldsymbol{x})$ defined over the whole domain by interpolation between the existing data [12]. The approximation as a linear combination of a radially symmetric function can be written as

$$
\hat{f}(\boldsymbol{x}) \approx \sum_{i=1}^{N} \alpha_{i} g_{i}(\boldsymbol{x}),
$$

where $\alpha_{i}$ are interpolation coefficients, and $g_{i}(\boldsymbol{x})$ are radial basis functions. The interpolation fit is strongly influenced by the choice of the radial function. In this

Table 1. Analysed radial basis functions

\begin{tabular}{lc}
\hline \hline RBF & $g_{i}(\boldsymbol{r})^{*}$ \\
\hline Spline type & $\boldsymbol{r}^{n}, n$ is odd \\
Linear & $\boldsymbol{r}^{n}$ \\
Cubic spline & $\boldsymbol{r}^{3}$ \\
Multiquadric & $\sqrt{1+\boldsymbol{r}^{2}}$ \\
Gaussian & $e^{-\boldsymbol{r}}$ \\
\hline
\end{tabular}

${ }^{*} \boldsymbol{r}$ is Euclidean norm, $\boldsymbol{r}=\left\|\boldsymbol{x}-\boldsymbol{x}_{j}\right\|$. 
study examined basis functions are summarized in Table 1 . The radially symmetric functions are based on Euclidean distance $r$. Following the theory, RBF should show good fitting of both deterministic and stochastic responses.

\subsection{POD-RBF METAMODEL}

The combination of POD and RBF leads to metamodel presented by Buljak [12] which gives a continuous approximation of the system over the chosen design space. The snapshot matrix $\mathbf{X}$ is generated by input vectors for which the values of approximation function are known and are stored in the matrix $\mathbf{Y}$. After obtaining responses using a full numerical or mathematical model or physical experiments, POD basis $\boldsymbol{\Phi}$ can compute. The matrix $\boldsymbol{\Phi}$ is truncated keeping only $K$ columns (significant singular values) to compute the corresponding amplitude matrix:

$$
\overline{\mathbf{A}}=\overline{\boldsymbol{\Phi}}^{\mathrm{T}} \mathbf{X} .
$$

Using RBF interpolation method (examples see in Table 1) and collecting results in the matrix $\mathbf{G}$, the coefficients of interpolation can be calculated

$$
\mathbf{B}=\left(\mathbf{G}^{-1} \overline{\mathbf{A}}^{\mathrm{T}}\right)^{\mathrm{T}} .
$$

The general formula for the approximation of the arbitrary design parameter vector $\boldsymbol{x}$ can be obtained by

$$
\hat{\boldsymbol{y}}(\boldsymbol{x}) \approx \overline{\mathbf{\Phi}} \cdot \mathbf{B} \cdot g(\boldsymbol{x}),
$$

where $\hat{y}$ is the corresponding approximated value for the observed value $y$.

\subsection{RESPONSE SURFACE MODEL}

Constructing metamodel using RSM, the relationship between responses and inputs can approximate by polynomial models. Madsen et al. [13] conclude that due to the complexity of the numerical modelling the noise in CFD results is inevitable. Based on [14], the second-order polynomial (9) is used as the response surface because of its robustness to numerical noise.

$$
\hat{\boldsymbol{y}}(\boldsymbol{x})=\beta_{0}+\sum_{i=1}^{N} \beta_{i} x_{i}+\sum_{i=1}^{N-1} \sum_{j=i+1}^{N} \beta_{i j} x_{i} x_{j},
$$

where $\beta$ is the coefficient of the polynomial. The coefficients are found by minimizing the sum of squared errors using the ordinary least squares equations [15]. 


\subsection{MOdEL VALIDATION}

Two different metrics, $R^{2}$ and leave- $k$-out cross-validation, are used to provide an estimation of the metamodel accuracy. The coefficient of determination, $R^{2}$ is used to set the fit of the metamodel. The $R^{2}$ is obtained as

$$
R^{2}=1-\frac{\sum\left(\boldsymbol{y}_{i}-\hat{y}_{i}\right)^{2}}{\sum\left(\boldsymbol{y}_{i}-\bar{y}_{i}\right)^{2}}=1-\frac{\text { MSE }}{\text { Variance }},
$$

where $\bar{y}$ is the mean value of the observed responses $y$. MSE (Mean Square Error) represents the departure of the metamodel from the full model, the variance shows how irregular the problem is [14]. If the model perfectly predicts the responses, then $R^{2}=1$.

The leave- $k$-out cross-validation approach is applied to avoid additional full numerical computations for metamodel validation. Based on Meckesheimer et al. [16] study, $k=1$ is suggested for low-order and RBF metamodels. Kalnins et al. [17] described the sigma cross-validation percentage error $\sigma_{\mathrm{cr} \%}$ to avoid the overestimation of the prediction error

$$
\sigma_{\mathrm{cr} \%}=100 \% \frac{\sqrt{\frac{1}{N} \sum\left(\hat{y}_{-i}-\boldsymbol{y}_{i}\right)^{2}}}{\frac{1}{N} \sum \boldsymbol{y}_{i}},
$$

where $\hat{y}_{-i}$ is the predicted approximation without the sample point $i(i=1,2, \ldots, N)$. It is assumed that the metamodel has acceptable accuracy if the sigma cross-validation percentage error is less than $10 \%$.

\subsection{EXPERIMENTAL PLAN}

The Design of Experiment method is applied to select sampling points in the design space. The sampling methods can divide into two groups: classical and space-filling methods. The classical designs are developed for physical experiments in origin. As reported by Jin et al. [14], for deterministic computer analysis space-filling designs should be applied to process all areas of the design space equally.

For the RBF approach, the impact of sample size on average accuracy and robustness is the smallest compared to other approximation methods [14]. In this study, for static metamodels are chosen 20 sample points in the three-dimensional design space. For transient metamodel is used two-dimensional space with 20 training points.

To select sampling points the Latin hypercube sampling [18] is chosen. As mentioned by Sessarego et al. [1], the Latin hypercube sampling (LHS) provides a uniform distribution of points in the entire space and on projected axes. Furthermore, 
LHS can generate an experimental plan for any number of points. The mean square error is used as a space-filling criterion to increase the quality of the experimental plan.

\section{Full Numerical Modelling of the System Behaviour}

The two-dimensional CFD simulations are done to find the excitation forces and the TC vibration amplitudes and frequencies due to the water cross-flow. An open source CFD software OpenFOAM 2.4.x is used to solve differential equations numerically. An arbitrary Lagrangian-Eulerian framework which combines Lagrangian approach for solid parts and Eulerian formulation for fluid regions is utilized for modeling of the fluid-structure interaction process, which characterizes fluid dynamics, structural dynamics and mesh dynamics [6] in the coupled system.

\subsection{NUMERICAL IMPLEMENTATION}

The turbulent, incompressible water cross-flow in the cylinder bundle is modeled using unsteady Reynolds-Averaged Navier-Stokes (unsteady RANS) equations. As Reynolds number at channel inlet is $1.13 \times 10^{4}$, the turbulence should be considered. The following two-equation turbulence models are utilized: standard k-epsilon and k-omega SST.

PIMPLE algorithm realizes the pressure-velocity coupling for time-dependent simulations. The chosen time step corresponds to at least 500 calculation steps per oscillation period. In the literature, for example [19], the suggested resolution is 100 steps per cycle. Therefore, the time step could not affect the calculation results.

The TC is placed in the middle of the fourth row of the cylinder bundle. The pitchto-diameter ratio $(P / d)$ is 1.1 . The TC has two degrees-of-freedom: it can move only in an $x_{1}-x_{2}$ plane without rotational movement, see (1). The motion of the TC is modelled using OpenFOAM built-in six degree-of-freedom solver. The pimpleDyMFoam OpenFOAM solver is utilized for time-dependent simulations. The TC has two springs and two dashpots one for each $\left(x_{1}\right.$ and $\left.x_{2}\right)$ direction. The schematic representation of the analysed system is shown in Fig. 1. The characteristic of the TC vibrations is determined by three design parameters: TC mass, stiffness and damping coefficients.

The TC boundary represents the interface between the fluid and solid domain, see Fig. 1. The TC movements deform the mesh only in the fluid domain and at a certain distance from the interface. The ring-shaped area is defined around the TC to avoid the mesh deformation impact on calculation results due to the cell size changes in the boundary layer. In this ring area, the cells cannot be shrunk or expanded. The updated position of the moving mesh points is calculated by Laplace's equation modifying mesh after each time step. 


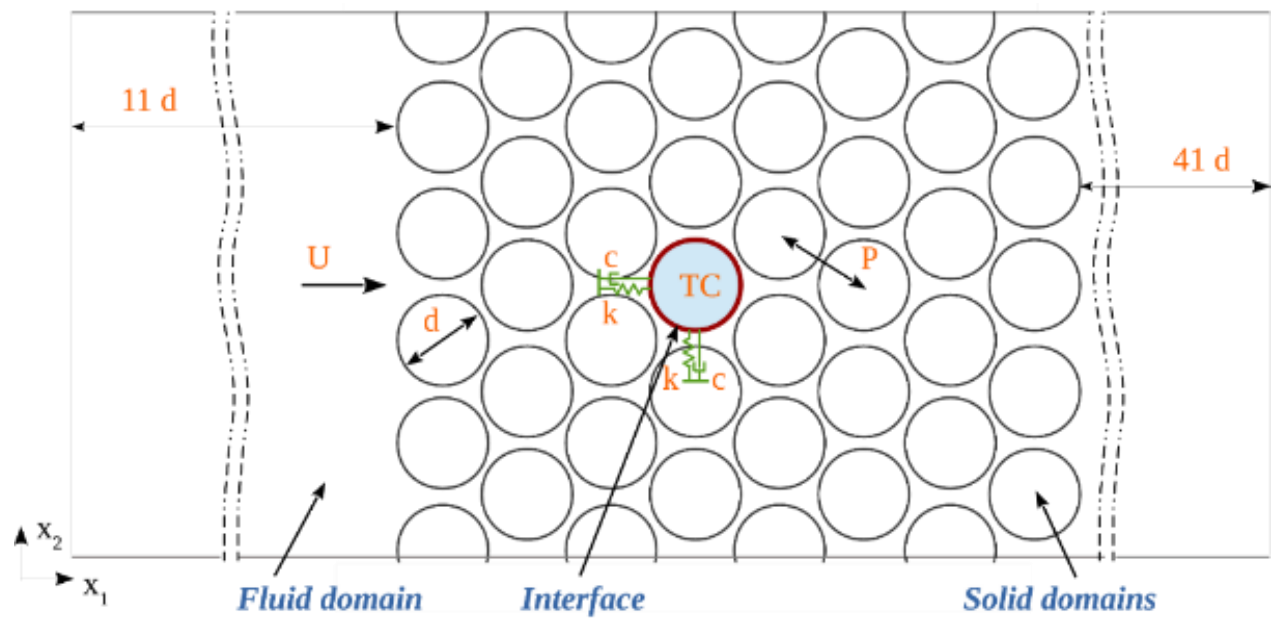

Fig. 1. Array configuration with a single, elastically-mounted cylinder TC, the direction of free stream flow $U$, the cylinder diameter $d$, the pitch $P$, the stiffness coefficient $k$ and the damping coefficient $c$.

\subsection{COMPUTATIONAL DOMAIN}

The Cartesian coordinate system is defined as follows: the $x_{1}$-axis points in the direction of the flow and the $x_{2}$-axis is in the perpendicular direction. The center of the $\mathrm{TC}$ is in the origin of the coordinate system.

Weaver and El-Kashlan [20] experimental results show that at least six rows are recommended in the tube array to study flow-induced vibrations. In this study, the computational domain contains cylinder bundle with 8 rows, therefore, can assume that the number of rows does not affect the TC behavior in the bundle. Each row contains five cylinders. The bundle is placed in rectangular channel 11 diameters of the TC after the inlet boundary and $41 d$ of the TC before the outlet boundary (see Fig. 1). The computational domain is discretized by quadrilateral cells. The maximum non-dimensional distance $y^{+}$from the wall to the first mesh node is from 12.5 till 41.5 .

\subsection{BOUNDARY AND INITIAL CONDITIONS}

The inflow water velocity $U$ is constant, at the middle of the channel it is $1.42 \mathrm{~m} / \mathrm{s}$. Constant pressure $\bar{p}$ is applied at the channel outlet. It is assumed that the turbulence intensity level in the channel is 5\%. The standard OpenFOAM wall functions are applied on the domain walls (cylinders and channel walls) for the turbulence kinetic energy $k_{t}$, the rate of dissipation of turbulence energy $\varepsilon_{t}$, the turbulent viscosity $\nu_{t}$ 
Table 2. Boundary conditions (BC)

\begin{tabular}{lccc}
\hline \hline Boundary name & Dirichlet BC & von Neumann BC & Wall functions \\
\hline Inlet & $U, k_{t}, \varepsilon_{t}, \omega$ & $\bar{p}$ & \\
Outlet & $\bar{p}$ & $U, k_{t}, \varepsilon_{t}, \omega$ & \\
Walls & $U$ & $\bar{p}$ & $k_{t}, \varepsilon_{t}, \omega$ \\
\hline
\end{tabular}

and the specific rate of dissipation $\omega$. Non-slip condition for velocity on the fixed cylinders and the channel top and bottom walls is used. The boundary conditions are summarized in Table 2.

The quasi-steady-state calculations in the fixed cylinder array with RANS approach have been done to obtain the initial conditions for the time-dependent simulations. During the RANS simulations, the impact of the main calculation parameters such as boundary conditions, turbulence models and cell size were studied as well. The quasi-static case was used for CFD model verification comparing modeled pressure drop in the channel with experimentally measured values [21]. The SIMPLE algorithm was applied to carry out the pressure-velocity coupling. Built-in OpenFOAM solver simpleFoam was used to solve differential equations.

\section{Results And Discussion}

\subsection{Response VALUES}

The LHS experimental plan determines 20 points in design space where the CFD results should be obtained with different combinations of design variables. The simulations were parallelized on the computational cluster due to the numerical complexity, the number of cases and time-consuming computations.

For the large amplitude vibrations (till $10 \%$ of the TC diameter, $d$ ), the input parameters are in the following ranges: $m \in$ [0.08957, 0.0989], $k \in[29.78 \times$ $\left.10^{4}, 32.91 \times 10^{4}\right], c \in[4.94,5.46]$. The lifetime of the system is assumed to be 10 oscillation periods.

The main vibrations are observed in the $x_{2}$-direction (perpendicular to the flow) which can be until 10-times larger than in $x_{1}$-direction in analysed cases. The movement of the TC creates an elliptic trajectory.

Small amplitude vibrations (till $0.05 \%$ of $d$ ) are modelled by the following ranges of design parameters: $m \in[0.1433,0.1575], k \in\left[49.75 \times 10^{3}, 55.28 \times 10^{3}\right], c \in$ $[38,42]$. The lifetime is three oscillation periods. Similarly, as in the large amplitude case, the dominant oscillations are perpendicular to the flow.

The output vectors contain the maximum amplitudes and the dominant frequencies in $x_{1}$ and $x_{2}$-direction when quasi-stationary oscillations are reached. The equi- 
librium point in $x_{1}$-direction is shifted toward the flow direction.

In average, the computation of one time-dependent case until a steady oscillation cycle takes approximately 20 days on 16 cluster cores.

\subsection{METAMOdEL WiTH STATIC INPUTS}

The POD-RBF approach is applied for the development of the static metamodel case to estimate the influence of each design parameter. The snapshot matrix $\mathbf{X}$ contains three design variables: mass, stiffness and damping coefficients at 20 sample points:

$$
\mathbf{X}=\left[\begin{array}{cccc}
m_{1} & m_{2} & \cdots & m_{20} \\
k_{1} & k_{2} & \cdots & k_{20} \\
c_{1} & c_{2} & \cdots & c_{20}
\end{array}\right]
$$

The damping and stiffness coefficients are equivalent in $x_{1}$ - and $x_{2}$-directions.

Corresponding responses: maximum amplitudes $A$ and dominant frequencies $f$ in both directions are collected in the matrix $\mathbf{Y}$

$$
\mathbf{Y}=\left[\begin{array}{cccc}
A_{x 1}^{1} & A_{x 1}^{2} & \cdots & A_{x 1}^{20} \\
A_{x 2}^{1} & A_{x 2}^{2} & \cdots & A_{x 2}^{20} \\
f_{x 1}^{1} & f_{x 1}^{2} & \cdots & f_{x 1}^{20} \\
f_{x 2}^{1} & f_{x 2}^{2} & \cdots & f_{x 2}^{20}
\end{array}\right] .
$$

The cross-validation percentage errors comparison of results from RBF metamodels with different basis functions is summarized in Table 3 and Table 4.

From Table 3 follows that large amplitude oscillations responses better predict multiquadric basis function, on average $\sigma_{\mathrm{cr} \%}=6.94 \%$. In cases with a linear and Gaussian approximations, the errors are around 10\%. The worst results show cubic spline basis function. All analysed basis functions have an error smaller than $10 \%$ except cubic spline in the case with small amplitude vibrations (see Table 4). The

Table 3. Comparison of cross-validation percentage error $\sigma_{c r} \%$ of metamodels with different radial basis functions (static metamodel, large amplitude vibrations)

\begin{tabular}{ccccc}
\hline \hline & Linear & Cubic spline & Gaussian & Multiquadric \\
\hline$A_{x 1}$ & $9.38 \%$ & $17.83 \%$ & $7.89 \%$ & $4.94 \%$ \\
$A_{x 2}$ & $12.05 \%$ & $16.26 \%$ & $8.75 \%$ & $8.07 \%$ \\
$f_{x 1}$ & $9.71 \%$ & $25.93 \%$ & $12.06 \%$ & $7.62 \%$ \\
$f_{x 2}$ & $9.79 \%$ & $22.31 \%$ & $11.56 \%$ & $7.15 \%$ \\
Mean & $10.23 \%$ & $20.58 \%$ & $10.07 \%$ & $6.94 \%$ \\
\hline
\end{tabular}


Table 4. Comparison of cross-validation percentage error $\sigma_{c r} \%$ of metamodels with different radial basis functions (static metamodel, small amplitude vibrations)

\begin{tabular}{ccccc}
\hline \hline & Linear & Cubic spline & Gaussian & Multiquadric \\
\hline$A_{x 1}$ & $8.15 \%$ & $15.99 \%$ & $8.58 \%$ & $24.39 \%$ \\
$A_{x 2}$ & $8.01 \%$ & $19.61 \%$ & $9.70 \%$ & $4.01 \%$ \\
$f_{x 1}$ & $7.61 \%$ & $17.79 \%$ & $8.63 \%$ & $2.52 \%$ \\
$f_{x 2}$ & $9.95 \%$ & $19.47 \%$ & $9.86 \%$ & $6.72 \%$ \\
Mean & $8.43 \%$ & $18.21 \%$ & $9.19 \%$ & $9.41 \%$ \\
\hline
\end{tabular}

multiquadric function gives the smallest errors except for the prediction of the amplitude in the flow direction. On average, the higher accuracy has linear approximation. RSM method gives very inaccurate approximation in investigated cases.

The design variable values are varied by $10 \%$ to estimate the influence of parameters on the results. The TC mass increasing leads to $12.4 \%$ decreasing in the dominant oscillation frequency. The mass influence is smaller on the TC maximum amplitudes; $-2.8 \%$ and $-8.8 \%$ depending on direction. The stiffness coefficient increasing mainly affects the maximum vibration amplitudes by $9.1 \%$ and $18.0 \%$ decreasing. The influence on dominant frequencies is non-negligible. The damping coefficient has the same impact on both the dominant frequencies and the maximum amplitudes decreasing values of about $5 \%$.

\subsection{Metamodel With Time-DEPENDENT INPUTS}

Dynamics of the system is investigated by the time-dependent metamodel developed by both RSM and POD-RBF techniques. In the transient case, the matrix $\mathbf{X}$ contains vectors with time-dependent excitation forces in both direction for 10 and 3 oscillation cycles depending on amplitude size:

$$
\boldsymbol{x}_{i}=\left[\left[F_{t 1}^{x 1} F_{t 2}^{x 1} \cdots F_{t n}^{x 1}\right],\left[F_{t 1}^{x 2} F_{t 2}^{x 2} \cdots F_{t n}^{x 2}\right]\right]^{\mathrm{T}} \quad i=1,2, \ldots, 20,
$$

where $n$ is the total number of uniformly distributed time samples.

As responses are saved the TC oscillation coordinates (in the plane) at corresponding time steps

$$
\boldsymbol{y}_{i}=\left[\left[x_{t 1}^{1} x_{t 2}^{1} \cdots x_{t n}^{1}\right],\left[x_{t 1}^{2} x_{t 2}^{2} \cdots x_{t n}^{2}\right]\right]^{\mathrm{T}}, \quad i=1,2, \ldots, 20 .
$$

The $R^{2}$ values are used to estimate time-dependent metamodels accuracy. The obtained results of large amplitude vibrations are summarized in Table 5. The average, the minimum and the maximum values of $R^{2}$ in the analyzed design space are presented. The RSM approximations give the very good results, in the worst case 
Table 5. $R^{2}$ values of different metamodels, large amplitude vibrations

\begin{tabular}{cccccc}
\hline \hline$R^{2}$ & Linear & Cubic spline & Gaussian & Multiquadric & RSM \\
\hline Average & 0.938 & 0.878 & 0.018 & 0.880 & 0.997 \\
Maximum & 0.998 & 0.999 & 0.077 & 0.995 & 0.999 \\
Minimum & 0.778 & 0.579 & 0.001 & 0.628 & 0.987 \\
\hline
\end{tabular}

$R^{2}=0.987$. From POD-RBF approach, the better behavior of the TC predicts the linear spline (on average $R^{2}=0.938$ ). The Gaussian approximation is not applicable for analysed situations. The comparison of typical approximations and the CFD simulation of the TC movement trajectories normalized by cylinder diameter $d$ are shown in Fig. 2. In the left graph, the metamodel is build using RSM with a second order-polynomial approximation. In the right side is POD-RBF model with linear spline approximation.
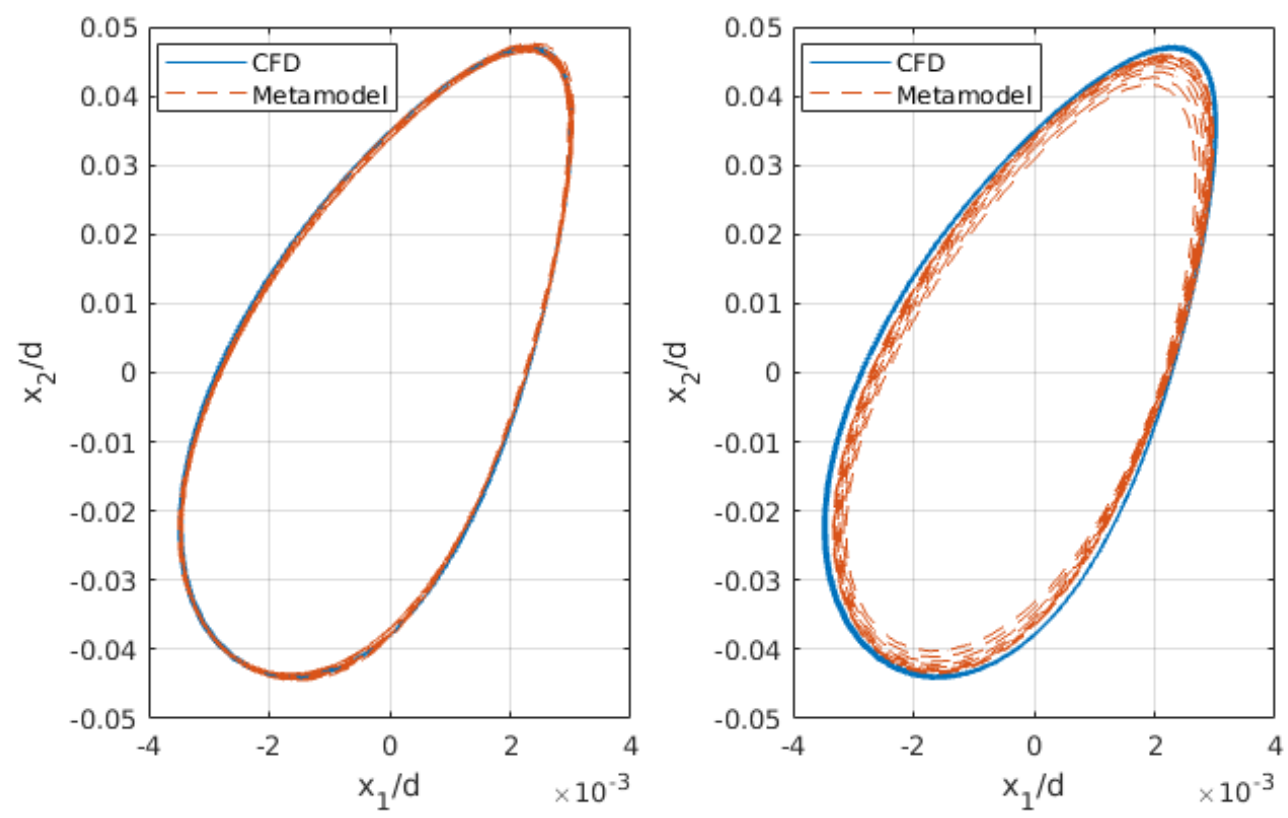

Fig. 2. Comparison of predicted and calculated normalized trajectories of the TC in the case of large amplitude vibrations. Left - RSM metamodel; right - POD-RBF with linear approximation.

Table 6 contains the corresponding $R^{2}$ values of small amplitude vibrations. It can be seen, that all chosen basis functions in small amplitude cases, normalized TC 
Table 6. $R^{2}$ values of different metamodels, small amplitude vibrations

\begin{tabular}{cccccc}
\hline \hline$R^{2}$ & Linear & Cubic spline & Gaussian & Multiquadric & RSM \\
\hline Average & 0.975 & 0.988 & 0.748 & 0.952 & 0.997 \\
Maximum & 1.0 & 1.0 & 0.999 & 1.0 & 1.0 \\
Minimum & 0.626 & 0.822 & 0.013 & 0.437 & 0.954 \\
\hline
\end{tabular}

trajectories predict better than large amplitude vibrations. There exist cases when $R^{2}>0.999$. At the same time, there are places in the design space where the accuracy is much lower, for example, $R^{2}=0.437$.

Figure 3 shows examples of normalized trajectories of small amplitude vibration. In the left side, the comparison of CFD and RSM metamodel is presented. The example of the cubic spline approximation is illustrated in the right graph.
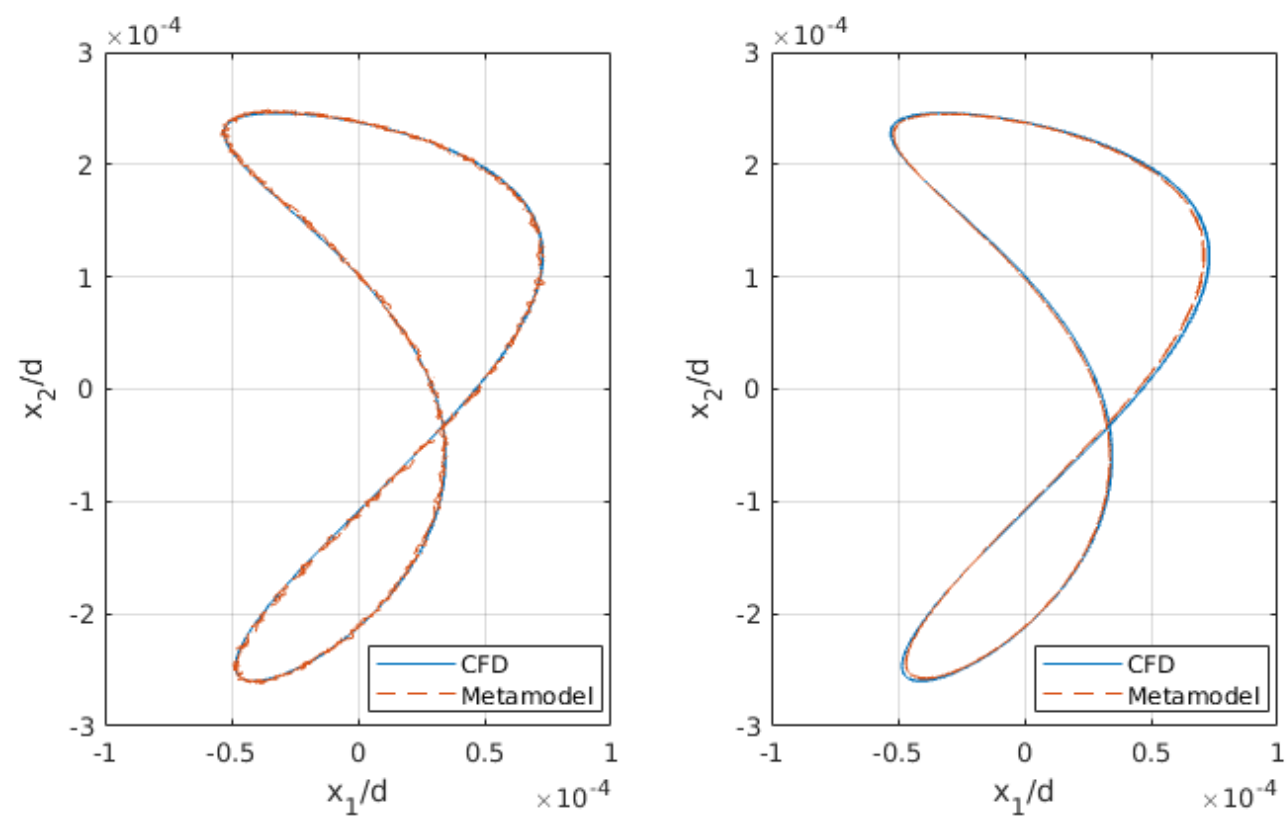

Fig. 3. Comparison of predicted and calculated normalized trajectories of the TC in the case of small amplitude vibrations. Left - RSM metamodel; right - POD-RBF with cubic spline approximation. 


\section{Conclusions}

The fast simulation tool is developed based on metamodeling approach to predict the behavior of the single elastically-mounted cylinder in the fixed cylinder array excited by water cross-flow. Two methodologies are applied to obtain the metamodels: Proper Orthogonal Decomposition-Radial Basis function with four different basis functions and Response Surface Method with a second-order polynomial approximation. The Computational Fluid Dynamics solvers are utilized to obtain the full model responses at given training points founded by Latin Hypercube Sampling plan. Three design parameters: the TC mass, stiffness and damping parameters are used to characterize the system. The values of parameters are varied by $10 \%$ to explore its influence on responses.

For the time-independent design variables (mass, stiffness and damping), the POD-RBF metamodels give better approximation comparing to the RSM metamodel. The cross-validation error of the responses (maximum amplitudes and dominant frequencies in both directions) for large amplitude vibrations of the TC with multiquadric basis function is $6.94 \%$. For small amplitude vibrations, the error is $8.43 \%$ using linear spline approximation. In both cases, the cubic spline gives the most significant error of $20.58 \%$ and $18.21 \%$ respectively.

The increasing of the TC mass by $10 \%$ leads to decreasing of the dominant oscillation frequency by $12.4 \%$. The mass influence on the TC maximum amplitudes is smaller: till $8.8 \%$. The stiffness coefficient mainly affects the maximum vibration amplitudes decreasing it by $9.1 \%$ and $18.0 \%$. The damping coefficient has the same impact on both the dominant frequencies and the maximum amplitudes (approximately $5 \%$ reduction).

The time history of the excitation force depending on the TC mass, stiffness and damping is used as input to predict the TC movement trajectory in time. It can be concluded that comparing the $R^{2}$ values the POD-RBF metamodels give unstable results in the analyzed design space. The coefficient of determination deviates very strongly depending on the point in the design space, for example from 0.013 to 0.999 . Using the RSM technique can achieve more stable predictions. All predicted cases are in the range between 0.954 and 1.0 .

The metamodeling is a robust approach to obtain a tool to predict the mass-springdamper system behavior in cross-flow-induced vibrations with acceptable accuracy, and it gives a significant reduction of the computational time and cost. For one timedependent CFD simulation is needed approximately 20 days on 16 cores computational cluster while metamodeling takes a few seconds on the desktop PC. For the future investigation, the time-dependent metamodeling should be evolved to obtain the fast simulation tool applicable to the monitoring of the system. 


\section{REFERENCES}

[1] M. Sessarego, N. Ramos-Garcia, H. Yang, W.Z. Shen (2016) Aerodynamic Wind-Turbine Rotor Design Using Surrogate Modeling and Three-Dimensional Viscous-Inviscid Interaction Technique. Renewable Energy 93 620-635.

[2] S. Gavrilin, S. SteEn (2017) Validation of Ship Manoeuvring Models Using Metamodels. Applied Ocean Research 66 178-184.

[3] A.-L. BraAtz, H. Hisken (2017) Response Surfaces for Advanced Consequence Models: Two Approaches. Journal of Loss Prevention in the Process Industries 49(B) 683-699.

[4] K. Khaledi, S. Miro, M. Konig, T. Schanz (2014) Robust and Reliable Metamodels for Mechanized Tunnel Simulations. Computers and Geotechnics 61 1-12.

[5] E. Mahmoudi, K. Khaledi, S. Miro, D. Konig, T. Schanz (2017) Probabilistic Analysis of a Rock Salt Cavern with Application to Energy Storage Systems. Rock Mechanics and Rock Engineering 50(1) 139-157.

[6] U.P.F. Filho, A.R.E. Antunes, S.M.A. Bastos, P.R.M. LyRA (2015) Minimization of Vortex Induced Vibrations Using Surrogate Based Optimization. Structural and Multidisciplinary Optimization 52(4) 717-735.

[7] Y.K. Son, G.J. SAVAGE (2018) A Simple Explicit Meta-model for Probabilistic Design of Dynamic Systems with Multiple Mixed Inputs. International Journal of Reliability, Quality and Safety Engineering 25(3) 1-14.

[8] H. Kato, K. FunazAKi (2014) POD-driven Adaptive Sampling for Efficient Surrogate Modeling and its Application to Supersonic Turbine Optimization. Proc. Of ASME Turbo Expo 2014: Turbine Technical Conference and Exposition, Dusseldorf, Germany, No. 2B, 1-10.

[9] S.U. HAMim, R.P. Singh (2017) Proper Orthogonal Decomposition-Radial Basis Function Surrogate Model-Based Inverse Analysis for Identifying Nonlinear Burgers Model Parameters from Nanoindentation Data. Journal of Engineering Materials and Technology 139(4) 0419010.

[10] Y.C. Liang, H.P. Lee, S.P. Lim, W.Z. Lin, K.H. LeE, C.G. Wu (2002) Proper Orthogonal Decomposition and its Applications - Part I: Theory. Journal of Sound and Vibration 252(3) 527-544.

[11] K. SimeK (2003) Properties of a Singular Value Decomposition Based Dynamical Model of Gene Expression Data. International Journal of Applied Mathematics and Computer Science 13(3) 337-345.

[12] V. BulJaK (2012) "Inverse Analysis with Model Reduction. Proper Orthogonal Decomposition in Structural Mechanics". Springer-Verlag, Berlin Heidelberg.

[13] J.I. Madsen, W. Shyy, T.R. HaftKa (2000) Response Surface Techniques for Diffuser Shape Optimization. AIAA Journal 38(9) 1512-1518.

[14] R. Jin, W. Chen, T. Simpson (2001) Comparative Studies of Metamodelling Techniques under Multiple Modelling Criteria. Structural and Multidisciplinary Optimization 23(1) 1-13. 
342 Modeling of the Elastically-Mounted Cylinder Behavior in the Cross-Flow

[15] T.S. Seecharan, G.J. Savage (2010) Performability-Based Design Optimization of Dynamic Systems. International Journal of Performability Engineering 6(2) 123-136.

[16] M. Meckesheimer, A.J. Booker, R.R. Barton, T.W. Simpson (2002) Computationally Inexpensive Metamodel Assessment Strategies. AIAA Journal 40(10) 20532060.

[17] K. Kalnins, J. Auzins, R.A. Rikards (2007) Fast Simulation Procedure for Ribbed Composite Structures with Material Degradation. Mechanics of Composite Materials 43(3) 225-232.

[18] P. Audze, V. Eglajs (1977) New Approach for Planning out of Experiments. Problems of Dynamics and Strengths 35 104-107 (in Russian).

[19] M. Hassan, A. Gerber, H. OMar (2010) Numerical Estimation of Fluidelastic Instability in Tube Array. Journal of Pressure Vessel Technology 132(4) 04130.

[20] D.S. Weaver, M. El-Kashlan (1981) On the Number of Tube Rows Required to Study Cross-Flow Induced Vibrations in Tube Banks. Journal of Sound and Vibration 75 265-273.

[21] S. Upnere, N. Jekabsons, S. Dementjevs, M. Wohlmuther (2018) Experimental Studies of a Single Flexibly-Mounted Rod in a Triangular Rod Bundle in CrossFlow. Proc. MATEC Web of Conferences 148 1-6. 